\title{
Research on the Influence of Phone Screen Color Combination on Visual Identification of the Aged
}

\author{
Delai Men ${ }^{*}$, Yulin Zhao \\ School of Design, South China University of Technology, Guangzhou, China \\ Email address: \\ 512080653@qq.com (Yulin Zhao), Mendelai@163.com (Delai Men) \\ ${ }^{*}$ Corresponding author
}

\section{To cite this article:}

Delai Men, Yulin Zhao. Research on the Influence of Phone Screen Color Combination on Visual Identification of the Aged. Humanities and Social Sciences. Vol. 4, No. 4, 2016, pp. 119-122. doi: 10.11648/j.hss.20160404.16

Received: June 20 2016; Accepted: July 16, 2016; Published: July 19, 2016

\begin{abstract}
With the development of mobile communications technology, smart phone has become a necessity in people's life. The aggravation of population aging in China draws the society's more attention to the market of smart phone for the aged. Being convenient, smart phone provides the aged with a brand new reading mode and a flexible reading environment. However, this advantage changes elderly users' habit of using eyes and causes visual fatigue. This research explores the relationship between smart phone screen color combination and visual identification, visual fatigue of the aged. Combining color criterion RGB adopted by most displayers with neutral colors black and white, the author probes into the influence of smart phone screen color combination on visual identification and visual fatigue through experiment. The experiment in this research is about the influence of mobile phone screen color combination on visual identification of the aged.
\end{abstract}

Keywords: Aged, Mobile Phone Screen, Color Combination, Identification

\section{Introduction}

Reading as one of the many important daily activities of older persons, not only brings to life more rich experience, at the same time can make older people reduce loneliness and enhance their confidence, slower mental decline. Now the people of more than 40 years old, they have the habit to reading on Smartphones and take it as their hobby, even dependent on the Smartphone screens. Among the older people in the future, they have the ability to grasp and learn the smart phone and they has the foundation for Smartphone use.

According to the research on color perception and coordination color mentioned in the study for the stimulation of human impact much larger than the object form, so the excellent color combinations could to help extend the older person operating time and the use of Visual performance and concentration. Therefore, good color vision of consciousness on the Smartphone screen, users can have a better reading experience. This study combines the elderly ocular physiological changes, using smart phone screens, and experiments to investigate the screen color combinations and depending on the relationship of consciousness of the elderly, research helps to guide the design of future products, Visual elements, reference to product design, care of the elderly and so on.

\section{Literature Review}

The aim of this article is to study the Smartphone screen color combination with the Visual identity of the elderly associated with the Visual fatigue, literature reviews are divided into seniors with vision theory, color theory, Visual recognition, Visual fatigue related literature, Visual performance of five for the collecting and analyzing to extend help in this study.

\subsection{Elderly and Visual Theory}

\subsubsection{Elderly}

For the definition of the elderly, gerontology in China and outside China nation has more than 10 kinds of opinions, one of the more popular are: to define, according to the physiological age to chronological age definition, defined by mental age definition age and society. Daily life in the "old" is usually for people of a certain age, so, in order to facilitate research and understanding, we divided by age age method citizen aged 60 . 


\subsubsection{Aging}

According to the United Nations standards, in a country or territory, population over 60 years of $10 \%$ or $7 \%$ of the population over the age of 65 , that is called in this country or region into an aging society. The ageing of the population, is a common problem all over the world.

\subsubsection{Visual Changes in the Elderly}

By the degradation of the organ after the age of 60 have more obvious. Under normal circumstances, people between ages 40 to 45 years old Visual deterioration, sharp decrease to about 65 years old can restore stability, in addition to the deterioration of vision in addition to capture the dynamic effects of fuzzy than before, the light shades of feeling will fade, lower sensitivity to color a lot.

Kline and Scialfa had proposed in 1996 population of light-sensitive, so we cannot adapt to the rapid changes in brightness observed close things begin to weakens; Cerella in 1985, narrows the view of older persons, the width of the field has been weakened.

Delai Men in 2013 in his studies of cognitive preferences that, based on the cognitive experience of the elderly, the elderly in most cognitive of tonal pleasure when, to order of red, Orange, yellow, blue, green and purple, the preference for the highest purity for purity of color, in the above should be used on the lightness of lightness.

The existence of these problems and widespread in the elderly population, the elderly in the Visual changes will be the Smartphone screen color combinations on the Visual identity of the elderly associated references and theoretical basis.

\subsection{Application of Color System}

\subsubsection{Color Formation}

Lin Kunfan mentioned in the color theory, when exposed to sunlight, survive light reflected to the eye as seen in the color of an object, light wave length difference, which produced red, Orange, yellow, green, blue and purple color vision. In the retina, with three types of cone-shaped like the Visual cells can sense that the 380 780nm light (the range of visible light). Color differences on the formation of color other than purely physical, another level, also have a psychological dimension, which contains subjective factors such as cognition, emotion, affect people of color feel.

\subsubsection{R, G, B Color Model}

$\mathrm{R}, \mathrm{g}$, b color model also known as additive color models, color comes from the red, green, and blue base color (RGB) brightness differences between overlapping, it is called additive color model. Model, red, green, and blue three base colours with 256 different values measured, the value from 0 to 255 , consists of three different combinations of values, forming a colorful color space. When $\mathrm{r}$ is $255, \mathrm{~g}$, $\mathrm{b}$ value of 0 is rendered in solid color red when $\mathrm{g}$ is $255, \mathrm{r}$ and $\mathrm{b}$ values are 0 for solid green; when the $\mathrm{b}$ value is $255, \mathrm{r}$, $\mathrm{g}$ is 0 , which is solid blue. $R, g, b$ values are all 0 values, to solid black. $R$, $\mathrm{g}, \mathrm{b}$ are 255 , the colour rendering solid color white. This study sample color schemes, picking colors primary colors $r$, g, b samples of solid color in design. 1821-1894 hemuhezi made sense three primary colors from the Germans in three types of cone cells in the eye, such as red light mainly dominated by Red cones, use color for a color condition in itself. In addition, the composition monitor screen color mode, tri-color CRT from the screen, which also belongs to the light and shade of gray scale performance and full-color trip (formed by the combination of $r, \mathrm{~g}, \mathrm{~b}$ colors). Principles and monitor screens of eyes to distinguish colors, are the three primary colors of light $\mathrm{r}, \mathrm{g}, \mathrm{b}$ color model.

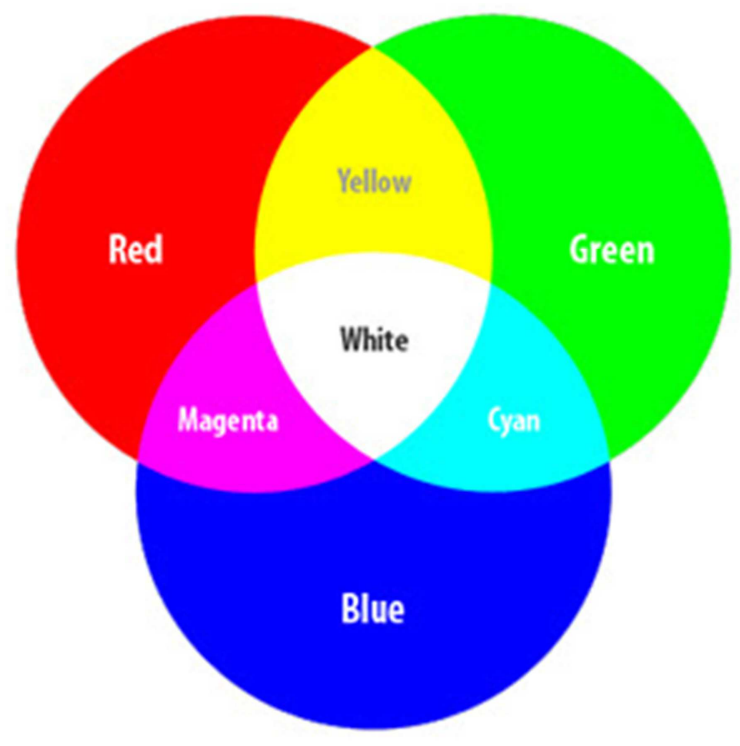

Figure 1. $R, G, B$ color model.

\subsection{Legibility}

\subsubsection{The Definition of Legibility}

Legibility, can be called easy reading, debate. Digit refers to can identify with each other which is why property, it affected by stroke, thickness, font, contrast and lighting, digit intervals, distances, influence of surrounding conditions such as white (Xu Tengxiong, and Peng You, and Wu Shuipi, 1991, human factors engineering, and cultural). Color vision of consciousness first depends on brightness difference, followed by color differences. Color combinations based on sample size and comparative view of consciousness is good or bad. Depending on the knowledge of high color, and other samples of the same size, degree of interpretation is the better. In Figure 2, for example, identification of blue-and-white than yellow background and blue-and-white view of consciousness than the yellow color scheme.

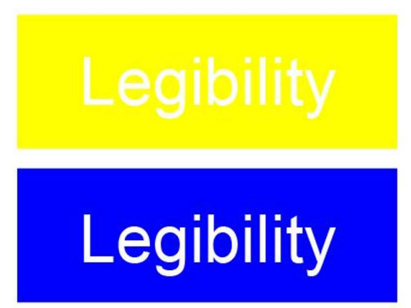

Figure 2. The difference of visual recognition of color combination. 


\subsubsection{Influence Factors of Visual Identity}

Ma Shicong (2002), Kaohsiung bus front road sign recognition study mentioned in the different diagrams, color in General is significantly affected, among the blue-and-white color scheme is the best cognitive and yellow background and red on a white background, white, and green knows the difference.

In addition, Ma Shicong (2002), Kaohsiung bus front destination tips mentioned in the cognitive study of P22-23, medium stroke thickness (thick higher than 1:6) digital sample of supposed to know better. Fine stroke thickness (thick higher than 1:8) followed by bold strokes (the rough higher than 1:5) the worst. For font construction also made the following conclusion, structural differences, the greater the font, read better, high similarity of composition, although obviously in design consistency, is likely to cause similar interpretation errors. In addition, Sun j Chambers (2007) digital reading render mode 15-29, found, for example, text size and also affect the interpretation of the form factor, and word-level and 14 for the new reading condition of thin out, the most appropriate.

\subsection{Visual Performance}

Lin Qingquan (2000), screen type, ambient lighting, and background color combinations for text terminals Visual task referred to in the study of the impact of Visual performance factors can be divided into three categories: (1) stimulating characteristics, (2) characteristics of the environment, (3) service features. In other words, Visual performance advantages and disadvantages of human external stimuli, human factors and environmental influences. This content contains two low lighting brightness factors such as ambient light and color matching to stimulate. Use of assessment methods in order to determine the advantages and disadvantages of Visual performance, increase the credibility of experimental objective.

\section{The Correlation Experiment of the Color Combination of the Smart Phone Screen on the Visual Recognition of the Elderly}

There is a close relationship between the factors that affect the vision of the elderly in the life. The ability of color identification provided by the eyes to improve the performance of reading for the elderly. This research will discuss the advantages and disadvantages of the eyes of the elderly for color combination, and the experiment will be based on the combination of color and color combination of the smart phone screen color combination of the elderly visual recognition of the relationship between the two. Due to the popularity of iPhone as well as the user's general praise, this experiment selected Apple's fifth generation mobile phone screen size 4 inches as a benchmark.

\begin{tabular}{|c|c|c|}
\hline Experimental objective & $\begin{array}{c}\text { Experimental } \\
\text { population }\end{array}$ & Experimental data \\
\hline & (1) $40 \mathrm{px}$ Visual performance of color \\
combination
\end{tabular}

Figure 3. Experimental architecture of this research.

(1) font design. Smart phone screen text, usually navigation main title of the font size for the $40 \mathrm{px}$, the main body size is $32 \mathrm{px}$, the vice text for the $26 \mathrm{px}$, small size is $20 \mathrm{px}$, it will be 40px, 32px, 26px, 20px as the experimental font size.

(2) color combination design. The design of the experimental samples, color selection color primaries in the $\mathrm{R}, \mathrm{G}$ and $\mathrm{B}$ color and color black and white five color cross match, formation of target and background combinations, and eliminates the color blind patients unable to discern the color combination, for a total of ten groups. For the blue bottom green words, at the end of the green blue words, white background black word, black bottom mispronounced character, white bottom blue words, blue bottom mispronounced character, at the end of the green black characters and black at the end of the green, red bottom mispronounced character, white at the bottom of the scarlet letter, respectively. Influence of object structure, consider the font structure, thickness and for visual knowledge factors and use e character in the history of Nailun e word scale, the strokes of the same thickness, spacing, text structure. And in accordance with the direction of the $\mathrm{E}$ word opening, the lower, left and right four groups for change. The 10 groups of color samples were tested, and the targets were 4 groups of 40 groups.

(3) the choice of the subject. Choose 20 old people who have the habit of using smart phones, 10 men and 10 women, aged between 65 and 60 years old.

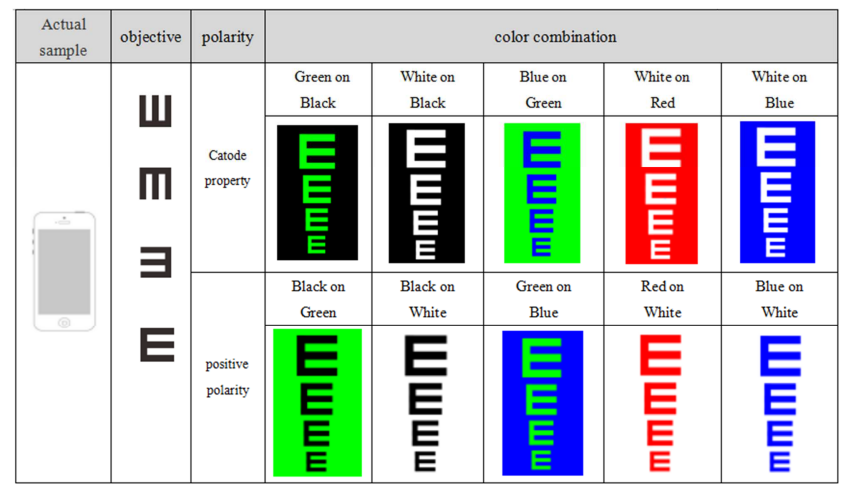

Figure 4. List of experimental color samples.

Visual performance based on the error rate of the subjects to answer the color of the sample to determine the relevance of the visual. The higher the error rate, the worse the visual recognition of the sample in the dark environment.

The actual number of Dacuo / actual item number of subjects with $* 100=$ error rate $(\%)$ 


\section{Conclusion}

\subsection{Color Sample Performance Ranking}

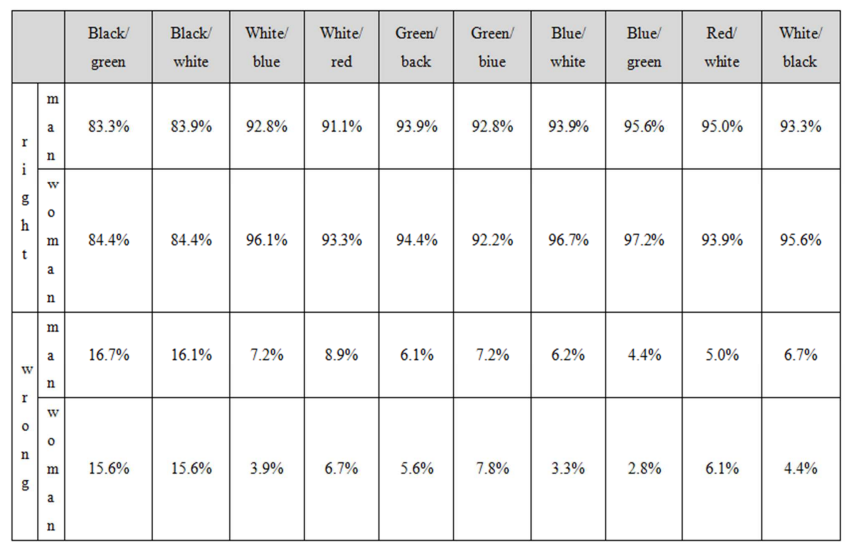

Figure 5. The list of error rate of colour sample.

\subsection{Analysis of the Performance of the Visual Recognition Performance of Color Samples}

Ranking (from superior to inferior):

Men: blue bottom mispronounced character $>$ red bottom wrongly written or mispronounced character $>$ blue bottom wrongly written or mispronounced character / at the end of the green $>$ black text on a white background black word $>$ white bottom blue word / at the end of the green blue word $>$ white bottom scarlet letter $>$ black bottom wrongly written or mispronounced character $>$ black bottom green

Women: blue bottom green word $>$ blue bottom wrongly written or mispronounced character $>$ white bottom blue word $>$ white bottom black word $>$ green black words $>$ red bottom wrongly written or mispronounced character $>$ white bottom scarlet letter $>$ green blue word $>$ black bottom wrongly written or mispronounced character / at the end of the black green

In this experiment, the subjects who answer right or wrong as discriminant as know the advantages and disadvantages of factors, the gender in the color sample of visual knowledge of bigger on, answer was 1.000; incorrect answers was 0.921 , both greater than 0.05 . There was no significant difference.

In the color combination as know the advantages and disadvantages of the performance, best male color samples for the bottom of the blue green word error rate $(4.4 \%)$, the worst color samples for black bottom green word error rate (16.7); best female color samples for the bottom of the blue green word error rate (2.8); the worst color sample is at the bottom of the dark green / black bottom mispronounced character error rate $(15.6 \%)$. Thus, the visual recognition of color samples, and not because of differences in gender factors.

\section{Acknowledgements}

Thanks to Delai Men in the Design Institute of South China University of Technology for my advice and guidance, he gave me a lot of advice on learning to help my research topics continue to improve and develop. His outstanding achievements in the field of his own profession and his persistent professional spirit is an example to me.

Due to the subject on the basis of limited, collection is not perfect, in order to achieve the optimum value of reliability and validity, the questionnaire before and after repeatedly revised several times, data investigation and numerous, coupled with elderly due to special groups, compared to the general population is very difficult, in looking for interview questionnaire survey pay a lot of effort, and I not talented, at this stage of the research is not deep enough, insufficiently is thorough exposition of ideas, it is inevitable mistakes and flaws in the Department, apologize, criticism and corrections also urge the experts, scholars and readers. Thus, thank you very much!

\section{References}

[1] Vatavu, Radu Daniel. Point \&click mediated interactions for large home entertainment displays [J]. Multimedia tools and applications. 2012(1): 113-128.

[2] Valenti R, Sebe N. What are you looking at [J]. International journal of computer vision.2012, 98(4): 324-334.

[3] Berman S, Stern H, Sensors for Gesture Recognition Systems [j]. IEEE Transactions on systems man and cybernetics part c-applications and reviews. 2012, 42(3): 277-290.

[4] Delai M, Dong W, Xiaoping H. The analysis and research of the smart phone's user interface based on Chinese elderly's cognitive character; 8th International Conference on Universal Access in Human-Computer Interaction, UAHCI 2014 [C] 2014, 138-146

[5] Lorenz, A. and Oppermann, [R]. 2009. Mobile Health Monitoring for the Elderly: Designing for Diversity. Pervasive and Mobile Computing 2009. 5: pp. 478-495

[6] Delai Men.A Study of cognitive behavior in relation to the elderly visual experience [C]. 7th International Conference on Universal Access in Human-Computer Interaction: Design Methods, Tools, and Interaction Techniques for eInclusion, UAHCI 2013, 2013, 594-603

[7] Murni, M., and Sri, H.K. "Involving psychometric tests for input device evaluation with older people. InOZCHI '05," [C] Proceedings of the 17th Australia conference on Computer-Human Interaction: Citizens Online: Considerations for Today and the Future, pp.1-10.2005

[8] Ute Ziegler. Multi-Sensory Design as a Health Resource: Customizable, Individualized, and Stress-Regulating Spaces. Design Issues: Volume 31, Number 1 Winter 2015

[9] Thomas van Rompay, Paul Hekkert and Wim Muller. The bodily basis of product experience. Design Studies Vol 26 No.4 July 2005

[10] Kohei Sugawara, Rikio Maruta. A Novel Intuitive GUI Method for User-Friendly Operation [J]. Knowledge-Based Systems, 2009, (22): 235. 